\title{
Posterior reversible encephalopathy syndrome in children, epiphenomenon of a known or unknown disease
}

\author{
Andrea Maria Alexandre, ${ }^{\mathrm{a}, *}$, Giuseppe Napoli ${ }^{\mathrm{b}}$, Antioco Sanna ${ }^{\mathrm{b}}$, Chiara Leoni ${ }^{\mathrm{b}}$ and Maria Ruggiero ${ }^{\mathrm{b}}$ \\ ${ }^{a}$ Catholic University of Sacred Heart, A. Gemelli Hospital, Institute of Radiology, Rome, Italy \\ ${ }^{\mathrm{b}}$ M. Bufalini Hospital, Neuroradiology Department, AUSL Cesena, Cesena, Italy
}

Received 26 August 2013

Submitted 30 October 2013

Accepted 31 October 2013

\begin{abstract}
Posterior reversible encephalopathy syndrome, also known as reversible posterior leukoencephalopathy syndrome or reversible posterior cerebral edema syndrome, is a recently described disorder of the cerebrovascular autoregulation system with multiple etiologies, most of which cause acute hypertension. Its clinical symptoms include headache, decreased alertness, mental abnormalities such as confusion, diminished spontaneity of speech, and changed behavior, stupor, seizures, vomiting, and abnormalities of visual perception such as cortical blindness. Radiologic appearance consists in cortical/subcortical posterior cerebral artery territory lesions. We describe two cases of this disease in pediatric patients caused by different clinical conditions but supported by the same pathophysiology. Early recognition of posterior reversible encephalopathy syndrome as a complication during different diseases and therapies in childhood may facilitate precise diagnosis and appropriate treatment.
\end{abstract}

Keywords: Posterior reversible encephalopathy syndrome (PRES), PRES associated diseases, magnetic resonance imaging, child

\section{Introduction}

Reversible posterior leucoencephalopathy syndrome (PRES), first described by Hinchey et al. [1], in 1996, is a neurological condition characterized by headache, nausea, vomiting, visual field disturbances, altered mental status, decreased alertness, and seizures. Several risk factors have been associated with development of PRES, including: acute/subacute systemic or malignant hypertension, preeclampsia and eclampsia, uremic encephalopathies (acute glomerulonephritis, hemolytic uremic syndrome), drug toxicity, thrombotic thrombocytopenic

*Corresponding Author: Dr Andrea Maria Alexandre, Catholic University of Sacred Heart, A. Gemelli Hospital, Institute of Radiology, Rome, Italy. E-mail: andrea.alexandre@libero.it purpura, vasculitidis, chronic renal insufficiency, blood transfusion or intravenous immunoglobulin treatment and post-transplantation stage in liver disease [1-3]. We report two cases of this disease in pediatric patients, caused respectively by nephrotic syndrome and lymphoma, that lead to acute hypertension, loss of cerebrovascular autoregulation of posterior cerebral artery (PCA) territory and PRES.

\section{Case report}

\subsection{Case report}

A 6-year-old boy was admitted to San Marino Republic hospital because of seizures associated with 
generalized hypotonia, poor contact with external stimulus and focal clonic seizures (lasting some minutes). He was affected by chronic renal failure due to multidrug resistant nephrotic syndrome, first treated with peritoneal dialysis, then with renal transplantation (that was subsequently removed after an arterial thrombosis), and then actually with haemodialysis three times per week On admission he was unconscious, with stiff neck and his head turned to the left, trisma, binocular conjugate upward rotation, and hypotonic lower limbs. Blood pressure was 200/160 mmHg. He was then intubated and anti-hypertensive and anticonvulsive therapies were administrated (nifedipine, four drops and diazepam), with prompt seizure resolution. Head CT scan was negative, at which point he was transferred to our hospital.

He arrived in our hospital sedated with fentanyl and midazolam, while receiving continuous mechanical ventilation with $\mathrm{FIO}_{2} 35 \%$. He was afebrile, with $\mathrm{SaO}_{2}$ $100 \%$, a heart rate of $85 / \mathrm{min}$, and blood pressure of 141/112 mmHg. Electroencephalography and hemogasanalysis were within normal range, as were his coagulation tests; his weight was $15,3 \mathrm{~kg}$. The following day he had a brain Magnetic Resonance (MR), which revealed multiple cortical-subcortical areas of hyperintensity on T2-weighted and Fluid Attenuated Inversion Recovery (FLAIR) images; these were mainly located in PCA territory (Fig. 1), with a minimal involvement
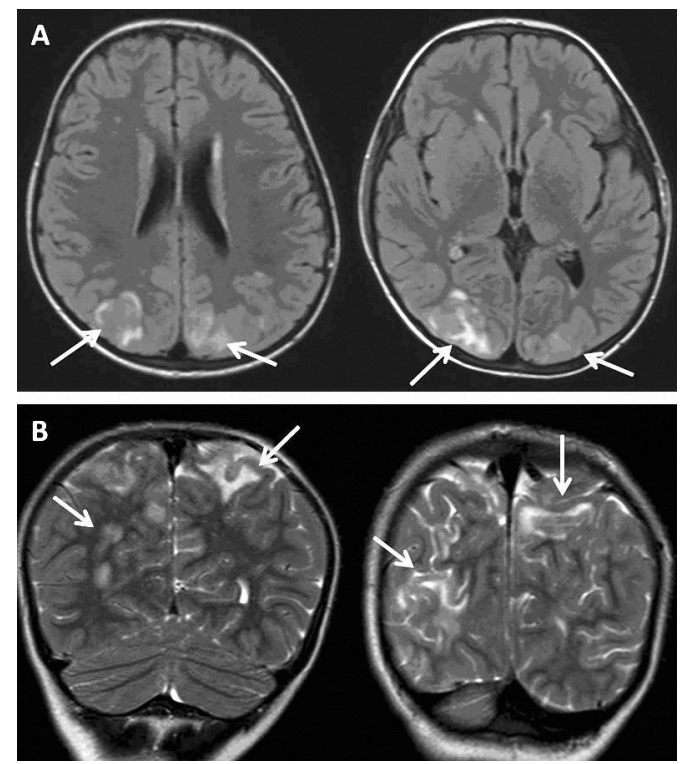

Fig. 1. Axial FLAIR (A) and coronal T2-weighted (B) images shows multiples cortical-subcortical areas of hyperintensity mainly located in PCA territory (arrows). of subcortical white matter of the frontal lobes and medulla oblongata (Fig. 2). Even if the latter are less frequent locations, MR findings were consistent with PRES.

Antihypertensive therapy was immediately administered with amlodipine ( $5 \mathrm{mg}$ once a day), ramipril ( 5 twice a day), atenolol ( $25 \mathrm{mg}$ once a day) and clonidine (50 mg thrice a day); hemodialysis was performed the same day. These treatments led to prompt resolution of his seizure episodes and normalization of his neurological examination.

\subsection{Case report}

A previously healthy, 11-year-old girl was admitted to our hospital because of progressive headache, vomiting, confusion and increasing abnormalities of visual perception (blurred vision), begining three days before admission.

On admission vital signs, and anthropometric measures were within normal limits, and she was conscious and reactive. Neurological examination confirmed bilateral visual reduction with normal fundus oculi, while the remainder of the neurological examination was unremarkable. She also had increased liver and spleen size, rhinolalia, and hypertension (170/110 mmHg).
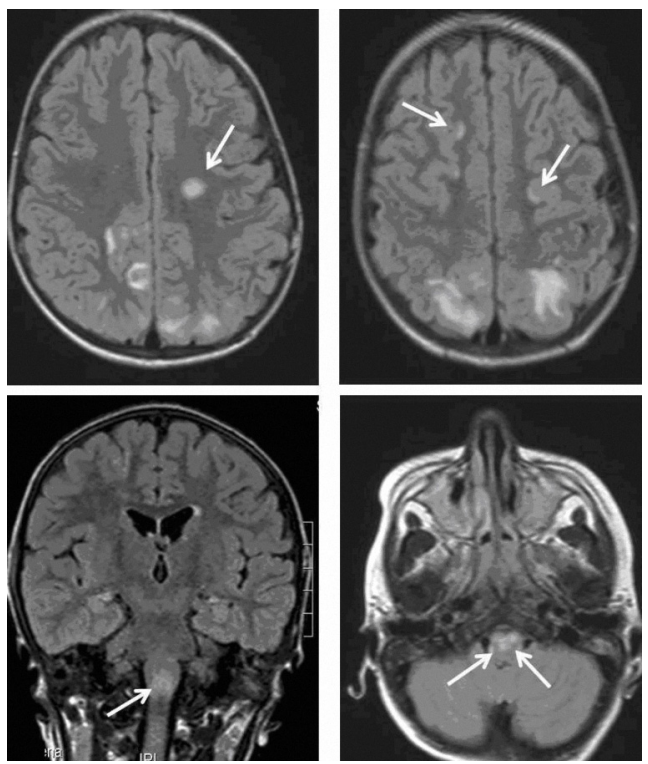

Fig. 2. Axial and coronal FLAIR images demonstrate a minimal involvement of subcortical white matter of frontal lobes and medulla oblongata (arrows). 
In laboratory studies, hemogram, serum electrolytes, metabolic screening of urine and blood, lactic acid, pyruvic acid, and liver function tests were normal. There was an increase in serum creatinine $(1,3 \mathrm{mg} / \mathrm{dl})$. Hemogasanalysis values were within normal ranges. Lumbar puncture demonstrated no white blood cells, and normal protein and glucose values. Cerebrospinal fluid and urine cultures were negative. Serologic tests in cerebrospinal fluid for EBV, HSV-1 and 2,VZV, CMV, HHV6, HHV-8 and Enterovirus RNA were negative.

Renal ultrasound revealed increased size of both kidneys, with some cortical hypoechogenic areas bilaterally. There was no stenosis of the renal artery on Doppler examination, but the resistivity index was increased bilaterally $(0,9)$.

Brain MR revealed substantial patchy symmrtrical cortical/subcortical lesions in parieto-occipital lobes (PCA territory), which appeared hyperintense on T2-weighted and FLAIR images, with restricted diffusion on Diffusion Weighted Images (DWI), and a normal Apparent Diffusion Coefficient (ADC) map, with a patchy enhancement on post-contrast T1-weighted images (Figs. 3 and 4). Clinical and MRI findings were consistent with PRES.

Based on these data, an anti-hypertensive therapy was begun (ramipril 5mg once a day and lecardip $10 \mathrm{mg}$ once a day), without a good control on pressure
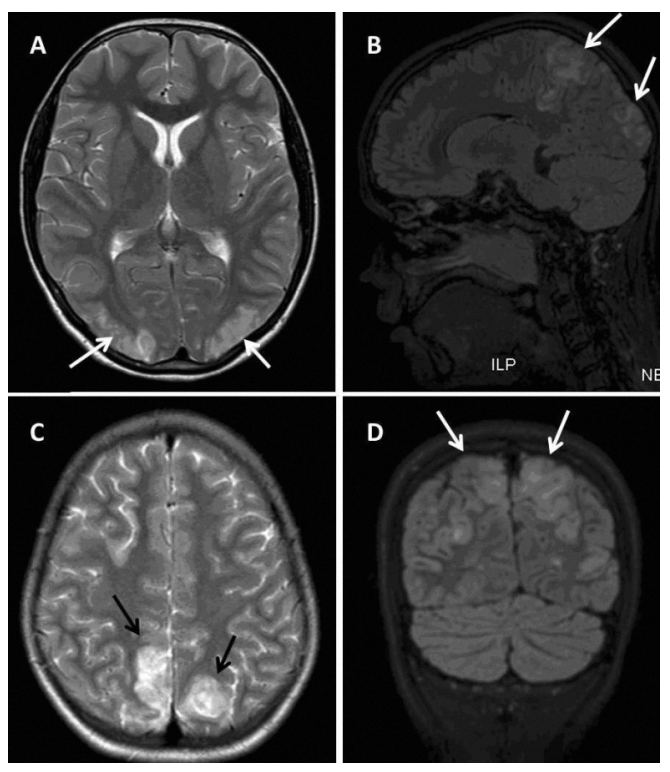

Fig. 3. Axial T2-weighted images (A and C), coronal and sagittal FLAIR images (B and D) revealed patchy cortical/subcortical substantially symmetrical lesions (arrows) in parieto-occipital lobes (PCA territory).
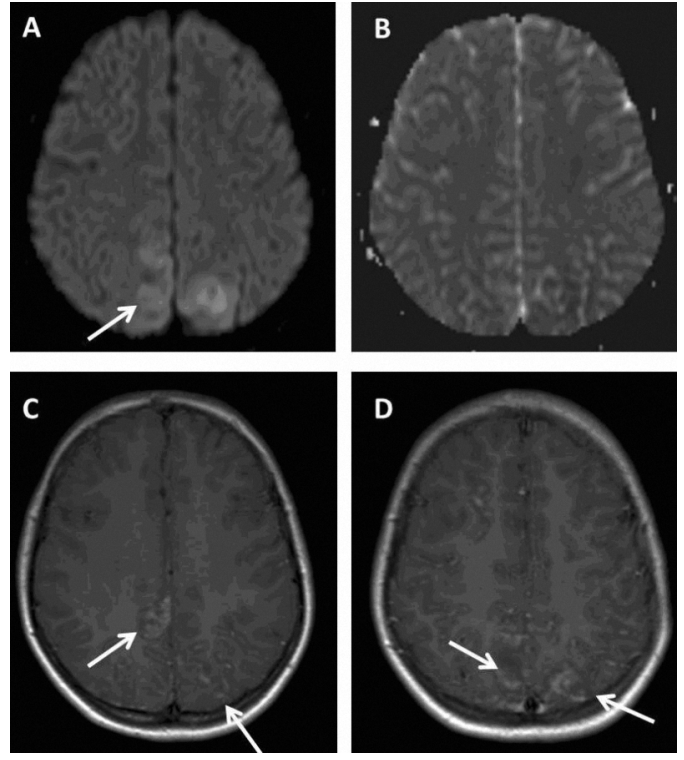

Fig. 4. The lesions shows restricted diffusion on DWI (arrow in A) and normal ADC map (B), with a pathcy enhancement on postcontrast T1-weighted images (arrows in $\mathrm{C}$ and D).

values $(140 / 80 \mathrm{mmHg})$. Furosemide, $40 \mathrm{mg}$ three times a day, was added to the therapy. There was a progressive reduction of diuresis and an increase of serum creatinine which increased to $4 \mathrm{mg} / \mathrm{dl}$. Clinical status of the young patient worsened with increasing epigastric pain and vomiting, while visual acuity increased form 20/50 to 20/25 (evaluated with Snellen chart) for both eyes. Renal biopsy was performed with the final diagnosis of non- Hodgkin lymphoma.

\section{Discussion}

PRES usually presents with seizure, headaches, cortical blindness and other visual abnormalities, altered mental status and focal neurologic signs, and it is frequently observed in patients with sudden increased blood pressure due to renal failure [4].

Several other conditions are reported to cause PRES, such as immunosuppressive (tacrolimus, cyclosporin A) and cytotoxic drugs, chemotherapeutic agents, fluid retention, eclampsia, IVIG treatment, sickle cell disease, systemic lupus erythematosus, polyarteritis nodosa, thrombotic thrombocytopenic purpura and hemolytic uremic syndrome [5-7].

Although the exact mechanism is not well understood, pathophysiology of PRES involves two main mechanisms [8]. Vasospasm, due to acutely increased 
blood pressure, and loss of autoregulation, are supposed to be possible factors. In the first theory, it has been suggested that loss of autoregulation of cerebral blood flow contributes to ischemia and cytotoxic cerebral edema. In the second theory, because of high blood pressure, temporary failure of autoregulatory capabilities of the cerebral vessels leads to arteriolar vasodilatation, endothelial dysfunction and hyperperfusion, and disrupted blood-brain barrier $[9,10]$.

Brain MR typically shows vasogenic edema within the occipital and parietal regions, usually symmetrical. Both cortical and subcortical locations are affected. Despite the name PRES, areas of vasogenic edema can have a non posterior distribution, mainly in watershed areas, including frontal, inferior temporal, cerebellar and brainstem regions. On CT images affected regions are hypodense while on MRI they are hypointense on T1-weighted images, hyperintense on T2-weighted and FLAIR images, with a patchy variable enhancement on post-contrast images [11].

Although the lesions are generally reversible with appropriate treatment, in unrecognized or not welltreated patients, the lesions can progress to ischemia and infarction; therefore, immediate diagnosis and treatment of hypertension is mandatory. In fact without rapid treatment, the functional vasogenic brain edema may cause cytotoxic brain edema, which causes permanent brain injury and neurologic sequelae $[12,13]$.

The initial evaluation should focus on rapid correction of blood pressure, preferably by parenterally administered anti-hypertensive drugs. Treatment, especially of neurological aspects of PRES are challenging. Some authors suggested continuous EEG monitoring in order to detect subtle or purely electrographic seizures [14]. Neurologists usually prefer traditional antiepileptic drugs (benzodiazepines, phenytoin or phenobarbital), even if benzodiazepines and phenobarbital may potentially worsen vigilance or cause respiratory depression, whereas phenytoin and fosphenytoin may induce adverse cardiac effects.

In the first case we report a known disease (chronic kidney disease caused by nephrotic syndrome) in a child with hypertension, in the second case we report an unknown disease (non- Hodgkin lymphoma) in another child with hypertension. In both cases immediate diagnosis and treatment of hypertension led to resolution of PRES induced neurological symptoms.

\section{Conclusion}

In pediatric patients with hypertension, seizure, headaches, visual abnormalities and typical PCA territory lesion on brain MRI we have to consider PRES among differential diagnosis. Increased blood pressure is thought to cause hyperperfusion with resultant vasogenic edema and endothelial damage. Prompt initiation of antihypertensive therapy may lead to reversal of the clinical and radiographic abnormalities of PRES [8]. Delay in diagnosis and therapy can result in permanent cerebral damage, while prompt recognition and immediate treatment are critical to avoid neurologic sequelae.

\section{References}

[1] Hinchey J, Chaves C, Appignani B, Breen J, Pao L, Wang A, et al. A reversible posterior leukoencephalopathy syndrome. N Engl J Med 1996;334(8):494-500.

[2] Van Diest D, Van Goethem JW, Vercruyssen A, Jadoul C, Cras P. Posterior reversible encephalopathy and GuillainBarré syndrome in a single patient: coincidence or causative relation? Clin Neurol Neurosurg 2007;109(1):58-62.

[3] Siebert E, Spors B, Bohner G, Endres M, Liman TG. Posterior reversible encephalopathy syndrome in children: radiological and clinical findings-a retrospective analysis of a German tertiary care center. Eur J Paediatr Neurol 2013;17(2): 169-75

[4] Ergün T, Lakadamyali H, Yilmaz A. Recurrent posterior reversible encephalopathy syndrome in a hypertensive patient with end-stage renal disease. Diagn Interv Radiol 2008;14(4): $182-5$.

[5] Onder AM, Lopez R, Teomete U, Francoeur D, Bhatia R, Knowbi O, et al. Posterior reversible encephalopathy syndrome in the pediatric renal population. Pediatr Nephrol 2007;22(11):1921-9.

[6] Servillo G, Bifulco F, De Robertis E, Piazza O, Striano P, Tortora F, et al. Posterior reversible encephalopathy syndrome in intensive care medicine. Intensive Care Med 2007;33(2):230-6.

[7] Aria R, Pisinski L. Posterior reversible encephalopathy syndrome. Pediatr Radiol 2010;40 Suppl 1:S171.

[8] Bartynski WS. Posterior reversible encephalopathy syndrome, part 2: controversies surrounding pathophysiology of vasogenic edema. AJNR Am J Neuroradiol 2008;29(6): 1043-9.

[9] Bartynski WS, Upadhyaya AR, Petropoulou KA, Boardman JF. Influenza A encephalopathy, cerebral vasculopathy, and posterior reversible encephalopathy syndrome: combined occurrence in a 3-year-old child. AJNR Am J Neuroradiol 2010;31(8): 1443-6.

[10] Roth C, Ferbert A. Posterior reversible encephalopathy syndrome: long-term follow-up. J Neurol Neurosurg Psychiatry 2010;81(7):773-7.

[11] Bartynski WS. Posterior reversible encephalopathy syndrome, part 1: fundamental imaging and clinical features. AJNR Am J Neuroradiol 2008;29(6):1036-42. 
[12] Prasad N, Gulati S, Gupta RK, Kumar R, Sharma K, Sharma RK. Is reversible posterior leukoencephalopathy with severe hypertension completely reversible in all patients? Pediatr Nephrol 2003;18(11):1161-6.

[13] Gümüş H, Per H, Kumandaş S, Yikilmaz A. Reversible posterior leukoencephalopathy syndrome in childhood: report of nine cases and review of the literature. Neurol Sci 2010;31(2): 125-31.

[14] Striano P, Striano S, Tortora F, De Robertis E, Palumbo D, Elefante A, et al. Clinical spectrum and critical care management of Posterior Reversible Encephalopathy Syndrome (PRES). Med Sci Monit 2005;11(11):CR549-53. 